
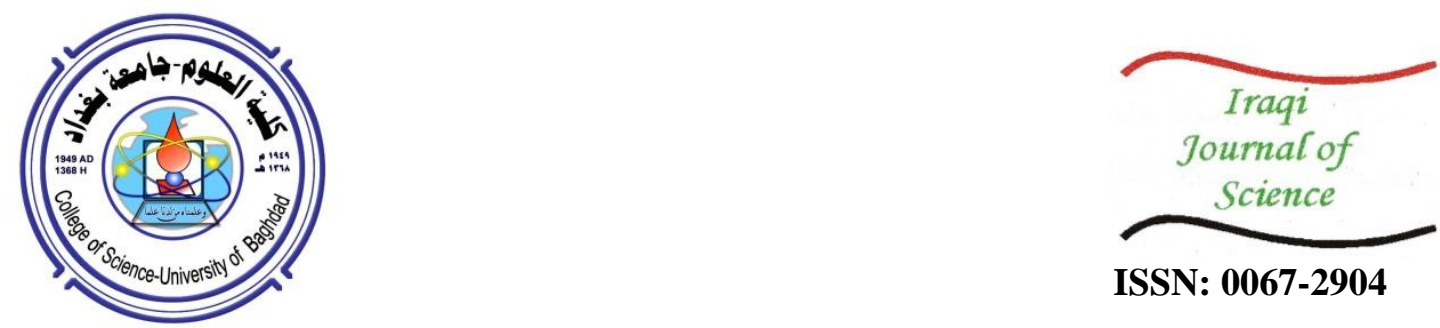

ISSN: 0067-2904

\title{
Numerical Solutions for the Optimal Control Governing by Variable Coefficients Nonlinear Hyperbolic Boundary Value Problem Using the Gradient Projection, Gradient and Frank Wolfe Methods
}

\author{
Jamil A. Ali Al-Hawasy*, Eman H. Mukhalef Al-Rawdanee \\ Department of Mathematics, College of Science, Mustansiriyah University, Baghdad, Iraq \\ Received: 11/12/ 2019 \\ Accepted: 15/3/2020

\begin{abstract}
This paper is concerned with studying the numerical solution for the discrete classical optimal control problem (NSDCOCP) governed by a variable coefficients nonlinear hyperbolic boundary value problem (VCNLHBVP). The DSCOCP is solved by using the Galerkin finite element method (GFEM) for the space variable and implicit finite difference scheme (GFEM-IFDS) for the time variable to get the NS for the discrete weak form (DWF) and for the discrete adjoint weak form (DSAWF) While, the gradient projection method (GRPM), also called the gradient method (GRM), or the Frank Wolfe method (FRM) are used to minimize the discrete cost function (DCF) to find the DSCOC. Within these three methods, the Armijo step option (ARMSO) or the optimal step option (OPSO) are used to improve the discrete classical control (DSCC). Finally, some illustrative examples for the problem are given to show the accuracy and efficiency of the methods.
\end{abstract}

Keywords: Numerical Classical Optimal Control, Galerkin Finite Element Method, Gradient Method, Gradient Projection Method, Frank Wolfe Method.

\section{الحلول العددية لسيطرة امثلية تحكمها مسألة قيم حدودية من نوع القطع الزائد غير خطية ذات معاملات متغيرة باستخدام طريقة مسقط الانحدار ، طريقة الانحدار وطريقة فرانك فولف.

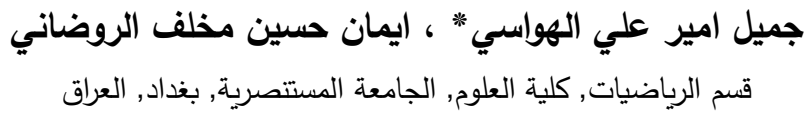

*Email: jhawassy17@uomustanriyah.edu.iq 
السيطرة التقليدية من النمط المقسم. في النهاية, تم إعطاء بعض الأمثلة العددية التوضيحية للمسألة لأظهار

$$
\text { دقة وكفاءة هذه الطرق. }
$$

\section{Introduction}

Optimization problems have wide applications in medicine, sciences and many other fields $[1,2]$. These applications are usually governed by partial differential equations (PDEs) or ordinary differential equations (ODEs).

Many researchers investigated the numerical solution of optimal control problems (NSOCPs) governed by nonlinear elliptic PDEs [3], semilinear parabolic PDEs [4], one dimensional linear hyperbolic PDEs with constant coefficients(LHPDES) [5], two dimensional linear and nonlinear hyperbolic PDEs with constant coefficients [6-9], two dimensional linear hyperbolic PDEs but with variable coefficients [10], or by one dimensional nonlinear ODEs [11]. The outcomes of these works have driven us to focus our interest on investigating the NSDCOC governed by the VCNLHBVP.

In this paper, the continuous classical optimal control problem (COCOCP) described by the VCNLHBVP is discretized by applying the Galerkin finite element method (GFEM) for the space variable and implicit finite difference scheme (GFEM-IFDS) for the time variable to get the DSCOCP ((the discrete weak form (DWF) for the VCNLHBVP and the discrete cost function (DGF)). To find such solutions, we should discuss the existence and the uniqueness theorem for the NS for the DWF. The proof of the existence theorem for the discrete classical optimal control (DCOC) and the necessary conditions of the problem are studied in a previous article [9] and they are all needed here. On the other hand, the DSCOCP is found numerically by using the GFEM-IFDS to find the NS of the DWF and then the DCOC by solving the optimization problem (the minimum of DCF) by using, separately, each one of the optimization methods; the GM, the GPM and the FWM. Within these three methods, the ARSO or the OPSO are used ,separately, to get better direction of the optimal search. Some illustrative examples for this problem are given to show the performance of each of these methods.

\section{Statement of the COCOCP [6]}

Let $\mathrm{K} \subset \mathbb{R}^{2}$ be a bounded open region, with boundary $\partial K$, and let $E=[0, T], 0<\mathrm{T}<\infty$ be a time space. The COCOCP governed by the VCNLHBVP, with control $\omega=\omega(\vec{x}, t) \in L^{2}(\rho)$ and state $\psi=$ $\psi_{\omega}(\vec{x}, t) \in H_{0}^{2}(\mathrm{~K})$, is

$$
\psi_{t t}-\chi \psi+\overline{\bar{a}}(\vec{x}, t) \psi=g(\vec{x}, \psi, t)+\omega-\omega_{d}, \forall(\vec{x}, t) \in \rho=\mathrm{K} \times \mathrm{E}, \vec{x}=(y, z)
$$

With the conditions

$$
\begin{aligned}
& \psi(\vec{x}, t)=0, \forall(\vec{x}, t) \in \partial \rho=\partial \mathrm{K} \times[0, T] \\
& \psi(\vec{x}, 0)=\psi^{0}(\vec{x}), \forall \vec{x} \in K \\
& \psi_{t}(\vec{x}, 0)=\psi^{1}(\vec{x}), \forall \vec{x} \in K
\end{aligned}
$$

where $\quad \chi=\sum_{\tau, \varrho=1}^{2} \frac{\partial}{\partial y_{\varrho}}\left[\bar{a}_{\varrho \tau}(\vec{x}, t) \frac{\partial}{\partial z_{\tau}}\right], \quad \overline{\bar{a}}(\vec{x}, t) \in L^{\infty}(K),(\forall \varrho, \tau=1,2) \quad$ are $\quad$ positive $\quad$ arbitrary functions, $\omega_{d}=\omega_{d}(\vec{x}, t) \in L^{2}(\rho)$ is a desired control and $g \in L^{2}(\rho)$ is a given function. The set of the admissible continuous classical controls is represented by $W_{a d}$, where $W_{a d}=\left\{\omega \in L^{2}(\rho) \mid \omega(\vec{x}, t) \in U\right.$, a.e. in $\left.\rho\right\}$, with $U \subset \mathbb{R}^{2}$ is a convex and compact set. The cost functional is defined by

$$
G_{0}(\omega)=\int_{\rho}\left[\frac{1}{2}\left(\psi-\psi_{d}\right)^{2}+\frac{\varpi}{2}\left(\omega-\omega_{d}\right)^{2}\right] d \vec{x} d t
$$

where $\psi_{d}=\psi_{d}(\vec{x}, t) \in L^{2}(\rho)$ is the desired state.

The CCOCP is to obtain $\omega \in \mathrm{W}_{a d}$ which minimizes equation (5).

Now, the weak form (WF) of the problems (1-4) for $\psi \in H_{0}^{1}(\mathrm{~K})$ is formulated by

$$
\begin{aligned}
& \left\langle\psi_{t t}, \varphi\right\rangle+B(t, \psi, \varphi)=(g(\vec{x}, \psi, t), \varphi)_{\mathrm{K}}+(\omega, \varphi)_{\mathrm{K}}-\left(\omega_{d}, \varphi\right)_{\mathrm{K}}, \forall \varphi \in \mathrm{S}=H_{0}^{1}(\mathrm{~K}) \\
& (\psi(0), \varphi)=\left(\psi^{0}, \varphi\right), \forall \vec{x} \in K \\
& \left(\psi_{t}(0), \varphi\right)=\left(\psi^{1}, \varphi\right), \forall \vec{x} \in K
\end{aligned}
$$

where $\psi^{0} \in \mathrm{S}, \psi^{1} \in L^{2}(\mathrm{~K})$ and the bilinear form is obtained by

$$
B(t, \psi, \varphi)=\sum_{\tau, \varrho=1}^{2}\left(\bar{a}_{\varrho \tau}(\vec{x}, t) \nabla \psi, \nabla \varphi\right)_{\mathrm{K}}+(\overline{\bar{a}}(\vec{x}, t) \psi, \varphi)_{K}
$$

Assumption A: For each $\varphi, \psi \in \mathrm{S}$ and $t \in E$, the following inequality is satisfied (I) $|B(t, \psi, \varphi)| \leq \sigma_{2}\|\psi\|_{1}\|\varphi\|_{1}$, where $\sigma_{1}>0$ 
(II) $|B(t, \varphi, \varphi)| \geq \sigma_{1}\|\varphi\|_{1}^{2}$, where $\sigma_{2}>0$.

Now, suppose that $\psi_{t}=\zeta$, then equalities (6-8) can be rewritten as follows

$$
\begin{aligned}
& \left\langle\zeta_{t}, \varphi\right\rangle+B(t, \psi, \varphi)=(g(\vec{x}, \psi, t), \varphi)_{\mathrm{K}}+(\omega, \psi)_{\mathrm{K}}-\left(\omega_{d}, \psi\right)_{\mathrm{K}}, \forall \varphi \in \mathrm{S} \\
& \left\langle\psi_{t}, \varphi\right\rangle=\langle\zeta, \varphi\rangle \\
& \left(\psi_{0}, \varphi\right)=\left(\psi^{0}, \varphi\right), \forall \vec{x} \in K \\
& \left.\left(\zeta_{0}, \varphi\right)=\left(\psi^{1}, \varphi\right),\right), \forall \vec{x} \in K
\end{aligned}
$$

3. Statement of the DSCOCP [6]: The COCOCP is discretized by applying the GFEM as follows: First, consider $B(., ., t)$ is dependent of $\mathrm{t}$, the region $\mathrm{K}$ can be divided into subregions (a polyhedron) for every integer (s), and $Z_{i}^{s}, i=1, \ldots, n$ be an admissible regular triangulation of $\overline{\mathrm{K}}$ i.e. $\overline{\mathrm{K}}=\bigcup_{i=1}^{n} Z_{i}^{s}$. Second, let $E_{j}^{S}=\left[t_{j}^{S}, t_{j+1}^{S}\right]$ be a subdivision of the interval $E$ and for $j=0,1, \ldots, m-1$, where each interval has a same length $\left(\Delta t=\frac{T}{m}\right.$ ). Let $S_{S} \subset S$ be the space of continuous piecewise affine mapping (CPAM) in $K$. The set of admissible discrete classical controls (DCC) is $W_{a d}^{s}=\left\{\bar{\omega}=\bar{\omega}^{s} \in W_{a d} \mid \bar{\omega}(\vec{x}, t)=\bar{\omega}_{i j} \in U^{s}\right.$ in $\left.\rho_{i j}\right\}$, where $\rho_{i j}:=Z_{i}^{s} \times E_{j}^{s}$

Now, $\forall \varphi \in S_{s}$, and for $j=0,1, \ldots, m-1$, the DWF of (9-12) can be obtained by

$$
\begin{aligned}
& \left(\zeta_{j+1}^{s}-\zeta_{j}^{s}, \varphi\right)_{\mathrm{K}}+\Delta t B\left(\psi_{j+1}^{s}, \varphi\right)=\Delta t\left(g\left(t_{j}^{s}, \psi_{j+1}^{s}\right), \varphi\right)_{\mathrm{K}}+\Delta \mathrm{t}\left(\omega_{j}^{s}-\omega_{d}\left(t_{j}^{s}\right), \varphi\right)_{\mathrm{K}} \\
& \left(\psi_{j+1}^{s}-\psi_{j}^{s}, \varphi\right)_{K}=\Delta t\left(\zeta_{j+1}^{s}, \varphi\right)_{K} \\
& \left(\psi_{0}^{s}, \varphi\right)_{\mathrm{K}}=\left(\psi^{0}, \varphi\right)_{\mathrm{K}} \\
& \left(\zeta_{0}^{S}, \varphi\right)_{\mathrm{K}}=\left(\psi^{1}, \varphi\right)_{\mathrm{K}}
\end{aligned}
$$

where $\psi_{j}^{S}=\psi\left(t_{j}^{S}\right), \zeta_{j}^{S}=\zeta\left(t_{j}^{S}\right) \in \mathrm{S}_{s}$ for $j=0,1, \ldots, m, \psi^{0} \in \mathrm{S}$, and $\psi^{1} \in L^{2}(\mathrm{~K})$.

The discrete cost functional (DCF) $G_{0}^{S}\left(\omega^{S}\right)$ is given by

$$
G_{0}^{S}\left(\omega^{s}\right)=\Delta t \sum_{j=0}^{m-1} \int_{\mathrm{K}}\left[\frac{1}{2}\left(\psi_{j+1}^{S}-\psi_{d}\left(t_{j+1}^{S}\right)\right)^{2}+\frac{\varpi}{2}\left(\omega_{j}^{S}-\omega_{d}\left(t_{j}^{S}\right)\right)^{2}\right] d \vec{x}
$$

Hence, the DSCOCP is to find $\bar{\omega}^{s} \in W_{a d}^{s}$, such that $G_{0}^{S}\left(\bar{\omega}^{s}\right)=\min _{\omega^{s} \in W_{a d}^{s}} G_{0}^{S}\left(\omega^{s}\right)$

Assumption (B): (I) Suppose that the function $g$ is defined on $Z_{i}^{S} \times E_{j}^{S} \times \mathbb{R}$ continuous with respect to $\psi_{j}^{s}$ and satisfies the following:

$\left|g\left(\vec{x}, t_{j}^{S}, \psi_{j+1}^{s}\right)\right| \leq H_{j}(\vec{x})+\alpha\left|\psi_{j+1}^{s}\right|$, for $j=0,1, \ldots, m-1, H_{j}(\vec{x})=H\left(\vec{x}, t_{j}^{s}\right) \in L^{2}(\mathrm{~K})$ and $\alpha \geq 0$.

(II) $\left|g\left(\vec{x}, t_{j}^{S}, \psi_{j+1}^{S}\right)-g\left(\vec{x}, t_{j}^{S}, \psi_{j}^{S}\right)\right| \leq C\left|\psi_{j+1}^{S}-\psi_{j}^{S}\right|$, for $(j=0,1, \ldots, m-1)$ and $C$ denotes the Lipschitz constant for any $j$.

3.1 Theorem (Existence and uniqueness of the DWF) [9]: For any fixed $j(0 \leq j \leq m-1)$, and for fixed DCC $\omega^{s} \in W_{a d}^{s}$, the DWF (13-16) has a unique solution $\psi_{\omega^{s}}^{s}=\psi^{s}=\left(\psi_{0}^{s}, \psi_{1}^{s}, \ldots, \psi_{m}^{s}\right)$, (for sufficiently small $\Delta t$ ).

\section{Existence of the DSCOCP:}

The following assumptions are useful to study the existence of the DCC.

Assumption C: The cost functional is of Caratheodary type, and satisfies :

$\left|\frac{1}{2}\left(\psi_{j+1}^{s}-\psi_{d}\left(t_{j+1}^{s}\right)\right)^{2}+\frac{\varpi}{2}\left(\omega_{j}^{s}-\omega_{d}\left(t_{j}^{s}\right)\right)^{2}\right| \leq \gamma_{j}^{s}(\vec{x})+\theta_{j}\left(\psi_{j}^{s}\right)^{2}, \forall j=0,1, \ldots, m-1$

where $\gamma_{j}^{S}(\vec{x})=\gamma^{s}\left(\vec{x}, t_{j}\right) \in L^{2}(\mathrm{~K})$ and $\theta_{j} \geq 0, \forall j=0,1, \ldots, m-1$.

The proofs of the following theorem and lemmas are shown in a previous article [9].

4.1 Theorem: The operator $\omega^{s} \mapsto \psi^{s}=\psi_{\omega^{s}}^{s}$ is continuous on $L^{2}(\mathrm{~K})$.

4.2 Lemma: The DCF is continuous with respect to (w.r.t.) the DCC on $L^{2}(\rho)$.

4.3 Lemma: If the DCC $\omega^{s}, \bar{\omega}^{s}$ are bounded in $L^{2}(\rho)$, and corresponding to the DSCCs $\omega_{j}^{s}$ and $\bar{\omega}_{j}^{S}=\omega_{j}^{S}+\Delta \omega_{j}^{S}$, then the discrete states are $\psi_{j}^{S}, \bar{\psi}_{j}^{s}=\psi_{j}^{S}+\Delta \psi_{j}^{S}$, respectively, then $(\forall j=$ $0,1, \ldots, m)$ :

$\left\|\Delta \psi_{h}^{s}\right\|_{1}^{2} \leq c\left\|\Delta \omega^{s}\right\|_{\rho}^{2}$ and $\left\|\Delta \zeta_{h}^{S}\right\|_{\mathrm{K}}^{2} \leq c\left\|\Delta \omega^{s}\right\|_{\rho}^{2}$

Or $\left\|\Delta \psi_{h}^{S}\right\|_{1}^{2} \leq \bar{c}$, and $\left\|\Delta \zeta_{h}^{S}\right\|_{\mathrm{K}}^{2} \leq \bar{c}$

\section{The Necessary conditions for DCCOCP}

The following theorem deals with the necessary conditions of the DCCOCP governed by VCNLHBVP. 
5.1 Theorem: Assume that DCF (17) is given and the DAWF (for the state equation) $\eta_{\omega^{s}}^{s}=\eta^{s}=$ $\left(\eta_{0}^{S}, \eta_{1}^{S}, \ldots, \eta_{m-1}^{s}\right)$ is given (for $\left.j=m-1, m-2, \ldots, 0\right)$ by

$$
\begin{gathered}
\left(\phi_{j+1}^{S}-\phi_{j}^{S}, \varphi\right)_{K}+\Delta t B\left(\eta_{j}^{s}, \varphi\right)=\Delta t\left(\eta_{j}^{s} g_{\psi_{j+1}^{s}}\left(t_{j}^{S}, \psi_{j+1}^{S}\right), \varphi\right)_{\mathrm{K}}+ \\
\Delta \mathrm{t}\left(\psi_{j+1}^{s}-\psi_{d}\left(t_{j+1}^{s}\right), \varphi\right)_{\mathrm{K}} \\
\eta_{j+1}^{s}-\eta_{j}^{s}=\Delta \mathrm{t} \phi_{j}^{s} \\
\eta_{m}^{S}=\phi_{m}^{S}=0
\end{gathered}
$$

where $\eta_{j}^{s}, \phi_{j}^{s} \in \mathrm{S}_{s}(\forall j=0,1, \ldots, m)$. Then the Fréchet derivative of DCF is given by

$$
\begin{aligned}
&\left(\mathrm{D} G_{0}^{s}\left(\omega^{s}\right), \omega^{\prime s}-\omega^{s}\right)=\Delta t \sum_{j=0}^{m-1}\left(H_{\omega_{j}^{s}}^{s}\left(t_{j}^{s}, \psi_{j+1}^{s}, \eta_{j}^{s}, \omega_{j}^{s}\right), \Delta \omega_{j}^{s}\right)_{\mathrm{K}} \\
&=\Delta t \sum_{j=0}^{m-1}\left(\eta_{j}^{s}+\varpi\left(\omega_{j}^{s}-\omega_{d}\left(t_{j}^{s}\right)\right), \Delta \omega_{j}^{s}\right)_{\mathrm{K}}
\end{aligned}
$$

where $\omega_{j}^{s}, \omega_{j}^{s} \in W_{a d}^{s}, \Delta \omega_{j}^{s}=\omega_{j}^{s}-\omega_{j}^{s}$ for $(j=0,1, \ldots, m)$, and $H^{s}$ is called the Hamiltonian. 5.1 Corollary: The inequality

$$
\Delta t \sum_{j=0}^{m-1}\left(\eta_{j}^{s}+\varpi\left(\omega_{j}^{s}-\omega_{d}\left(t_{j}^{s}\right)\right), \Delta \omega_{j}^{s}\right)_{\mathrm{K}} \geq 0, \forall \omega_{j}^{s} \in W_{a d}^{s}
$$

is equivalent with the minimum principle blockwise $\forall j=0,1, \ldots m-1$

$$
\left(\eta_{j}^{S}+\varpi\left(\omega_{j}^{S}-\omega_{d}\left(t_{j}^{S}\right)\right), \omega_{j}^{S}\right)_{T_{i}}=\min _{\omega^{s} \in W_{a d}^{s}}\left(\eta_{j}^{S}+\varpi\left(\omega_{j}^{S}-\omega_{d}\left(t_{j}^{S}\right)\right), \omega_{j}^{s}\right)_{T_{i}}
$$

\section{Main results (Solution methods):}

This section is devoted to present our method which is used to solve the DCCOC governed by the VCNLHBVP, the DWF (and the DAWF) are solved by using the mixed GFEM-IFDS, while the minimum values for the DCF and the DCOC are found by using, separately, each one of the GM, FWM, or GPM. Within each of these three methods, the ARSO and the OPSO are used, separately, to improve the value of the DCOC. The following algorithm shows the steps of this method in details.

6.1 ALGORITHIM: Let , $c \in(0,1),\left\{\delta^{s}\right\}$ be a sequence with $\delta^{s} \in(0, \infty)$, or $\delta^{s} \in(0,1]$, for each $s$. $\mu>0$. and let $\omega^{s} \in U$ be an initial control.

Step1: Set $s:=0$.

Step 2: Solve the DWF (13-16) (the DAWF (18-20)) by using GFEM-IFDS to get the state $\psi^{s}$ (the adjoint solution $\eta^{s}$ ), and then calculate $G\left(\omega^{s}\right)$ and $D G\left(\omega^{s}\right)$ from (17) and (21), respectively.

Step 3: Find a new direction (new control) $u^{s} \in U$ (i.e. a direction $u^{s}-\omega^{s}$ ), by applying the following methods, separately:

(a) GM: Find $u^{s} \in U$, such that: $\quad u^{s}=\omega^{s}-\frac{1}{\mu} D G\left(\omega^{s}\right)$

(b) FWM: Find $u^{s} \in U$, such that: $\left(D G\left(\omega^{s}\right), u^{s}-\omega^{s}\right)=\min _{u \in U}\left(D G\left(\omega^{s}\right), u-\omega^{s}\right)$

(c) GPM: Find $u^{s} \in U$, such that:

$$
\xi^{s}=\left(D G\left(\omega^{s}\right), u^{s}-\omega^{s}\right)+\frac{\mu}{2}\left\|u^{s}-\omega^{s}\right\|^{2}=\min _{u \in U}\left(D G\left(\omega^{s}\right), u-\omega^{s}\right)+\frac{\mu}{2}\left\|u-\omega^{s}\right\|^{2}
$$

Step 4: Solve the DWF (13-16) to find the state solution $\psi^{s}$ corresponding to the new $u^{s}$.

Step 5: Calculate $\xi^{s}=\left(D G\left(\omega^{s}\right), u^{s}-\omega^{s}\right),\left(\xi^{s}=-\frac{1}{\mu}\left\|D G\left(\omega^{s}\right)\right\|^{2} \mathrm{Z}\right.$ in the GM $)$

If $\xi^{s}=0$, then stop the process . (where $\|\cdot\|$ is the norm-2 with respect to vector space $Q$ ).

Step 6: Choose $\delta^{s}$ by using one of the following methods:

ARSO: Assume an initial value $\delta^{s} \in[0,+\infty)\left(\right.$ or $\left.\delta^{s} \in[0,1]\right)$. If $\delta^{s}$ satisfies the inequality

$$
\mathrm{X}_{s}\left(\delta^{s}\right)=G\left(\omega^{s}+\delta^{s}\left(u^{s}-\omega^{s}\right)\right)-G\left(\omega^{s}\right) \leq \delta^{s} b \xi^{s}
$$

we set $\delta:=\delta / c$, and choose the last $\delta \in(0, \infty)$ for $\delta^{s}$, that satisfies the above inequality. If not satisfied, we denote $\delta:=\delta c$, and choose for $\delta^{s}$ the first $\delta^{s} \in(0, \infty)$ (or $\delta^{s} \in(0,1]$ in GM) that satisfies the above inequality.

OPSO: Find an $\delta^{s} \in[0,1]$, such that: $\left(D G\left(\omega^{s}\right), u^{s}-\omega^{s}\right)=\min _{\delta^{s} \in[0,1] .}\left(D G\left(\omega^{s}\right), u-\omega^{s}\right)$

Step 7: Set $\omega^{s+1}=\omega^{s}+\delta\left(u^{s}-\omega^{s}\right), s:=s+1$ and we go to step 2 .

\section{Numerical examples}

This section contains some illustrative examples which show the activity of the methods which are 
given in algorithm (6.1). MATLAB software is used to achieve the above algorithm. The GFEMIFDS is used in step (2) to find the DS $\psi^{s}\left(\eta^{s}\right)$, with $n=9(h=0.1), m=20,(\Delta t=0.05)$. In the GM, GPM and FWM, the parameters take the values of $b=c=0.5$ and $\mu=0.5$.

7.1 Example: Consider the following COCOCP governed by the VCNLHBVP:

$$
\begin{aligned}
& \psi_{t t}-\frac{\partial}{\partial y}\left[e^{z} \frac{\partial \psi}{\partial y}\right]-\frac{\partial}{\partial z}\left[e^{y} \frac{\partial \psi}{\partial z}\right]+e^{\pi y-z} \psi=g(\vec{x}, t)+\sin (\psi)-\sin \left(\psi_{d}\right)+\omega-\omega_{d}, \quad \forall(\vec{x}, t) \in \rho, \\
& E=[0,1], \mathrm{K}=[0,1] \times[0,1], \& \vec{x}=(y, z) . \\
& \psi(\vec{x}, t)=0, \forall(\vec{x}, t) \in \partial \rho=\partial \mathrm{K} \times[0, T] . \\
& \psi(\vec{x}, 0)=0, \text { and } \psi_{t}(\vec{x}, 0)=0.75 y z\left(1-e^{z(1-z)}\right)(1-y), \quad \forall \vec{x} \in K \\
& \text { where } \quad g(\vec{x}, t)=\frac{3 z e^{z} \sin (t)\left(e^{(-z(z-1))-1}\right)}{2}-e^{y}\left(2 y e^{(-z(z-1))} \sin (t)(2 z-1)\left(\frac{3 * y}{4}-\frac{3}{4}\right)+\right. \\
& \left.2 y z e^{(-z(z-1))} \sin (t)\left(\frac{3 z}{4}-\frac{3}{4}\right)-y z e^{(-z(z-1))} \sin (t)(2 z-1)^{2}\left(\frac{3 y}{4}-\frac{3}{4}\right)\right)+y z \sin (t)\left(\frac{3 y}{4}-\right. \\
& \left.\frac{3}{4}\right)\left(e^{(-z(z-1))}-1\right) y z e^{(\pi y-z)} \sin (t)((3 y) / 4-3 / 4)\left(e^{(-z(z-1))}-1\right)
\end{aligned}
$$

The control constraint is $U=[-0.5,1]$ and the cost function (5) with

$\psi_{d}(\vec{x}, t)=0.75 y z(1-y)\left(1-e^{z(1-z)}\right) \sin (-t), \forall(\vec{x}, t) \in \rho$, and

$\omega_{d}(\vec{x}, t)=\left\{\begin{array}{cc}0.25 & , \text { for } 0 \leq t<0.5 \\ -0.25 & , \text { for } 0.5 \leq t \leq 1\end{array}\right.$

with the initial control

$\omega_{0}(\vec{x}, t)=0.35-\left(2 t e^{-t}\right), \forall(\vec{x}, t) \in \rho$.

First, depending on the above initial control and its corresponding state, the following results are obtained according to the optimization methods with ARSO .

(I) In the GM: the optimal control and the corresponding state are obtained after 11 iterations. The results are: $G_{0}\left(\omega^{s}\right)=4.6678 \mathrm{e}-06, \varrho_{s}=9.5 \mathrm{e}-03$, and $\varepsilon_{s}=1.1 \mathrm{e}-03$

where $\varrho_{s}$ and $\varepsilon_{s}$ are the discrete maximum errors for the state and control, respectively.

The optimal control and its corresponding state are obtained at $t=0.5$ by the following figures.

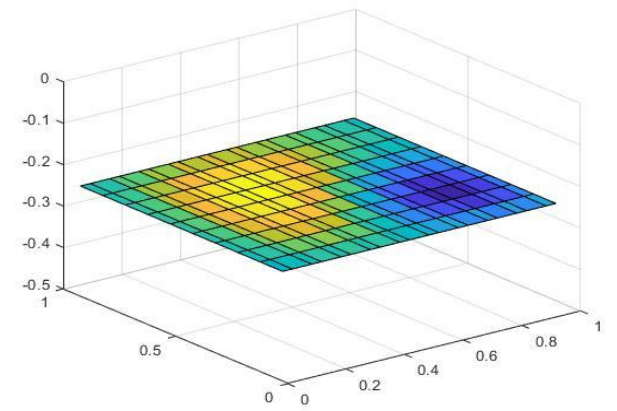

Figure 1- The optimal control

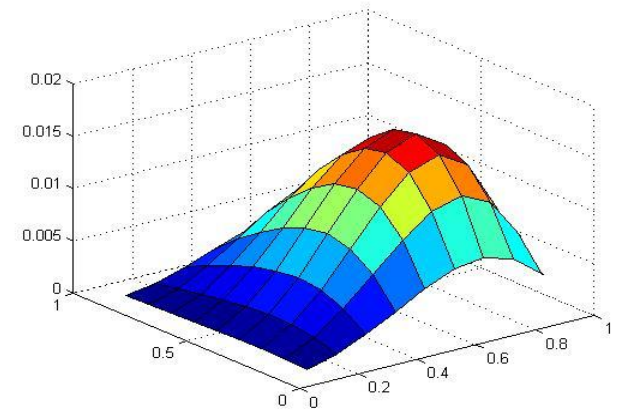

Figure 2- The Corresponding state

(II) In the FWM: the optimal control and corresponding state are showed after 117 iterations, the results are: $G_{0}\left(\omega^{S}\right)=7.0095 \mathrm{e}-06, \varrho_{s}=9.8 \mathrm{e}-03$, and $\varepsilon_{s}=9.9 \mathrm{e}-03$

The following figures show the optimal control and its corresponding state at $t=0.5$ :

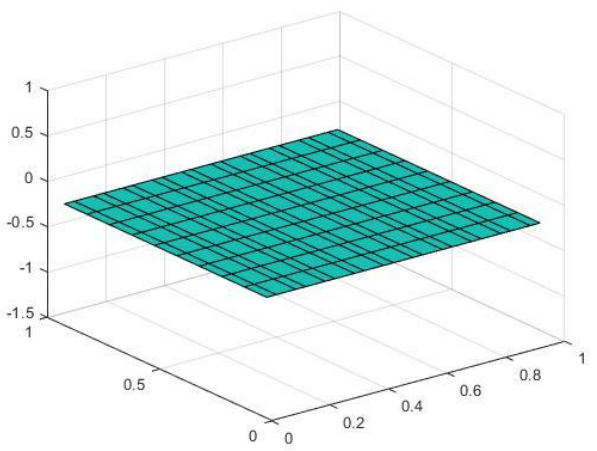

Figure 3- The optimal control

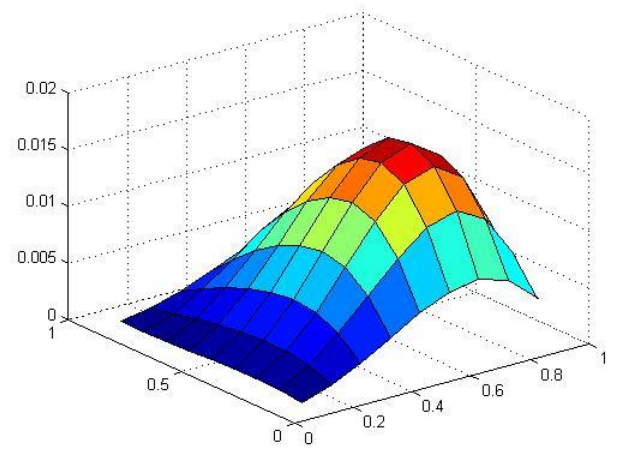

Figure 4- The Corresponding state 
(III) In the GPM: the optimal control and corresponding state are obtained after 4 iterations. The results are: $G_{0}\left(\omega^{S}\right)=4.6450 \mathrm{e}-06, \varrho_{s}=9.5 \mathrm{e}-03$, and $\varepsilon_{s}=7.7140 \mathrm{e}-04$

The following figures of this method are also shown at $t=0.5$.

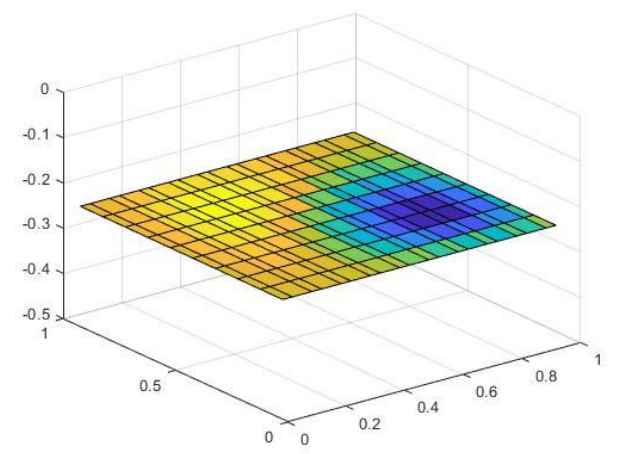

Figure 5- The optimal control

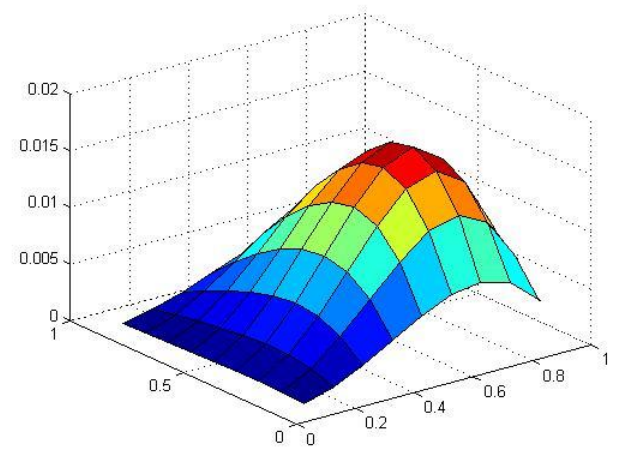

Figure 6- The Corresponding state

Second, the following results are obtained by using the optimization methods with OPSO:

(I) In the GM and GPM: the optimal control and corresponding state are given after 3 iterations

The results in this case are: $G_{0}\left(\omega^{s}\right)=4.5746 \mathrm{e}-06, \varrho_{s}=9.5 \mathrm{e}-03$, and $\varepsilon_{s}=1.5 \mathrm{e}-03$.

The optimal control and its corresponding state are shown at $t=0.5$ by the following figures.

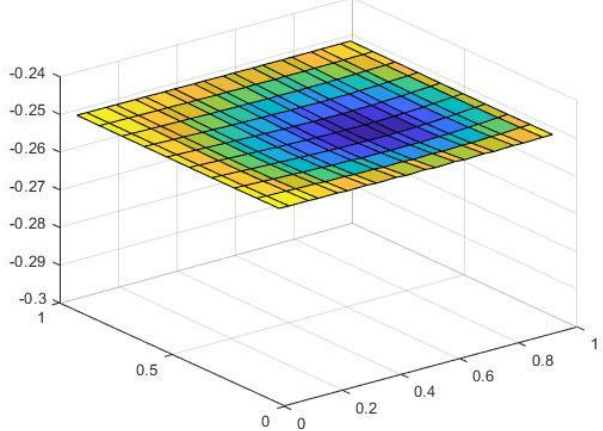

Figure 7- The optimal control

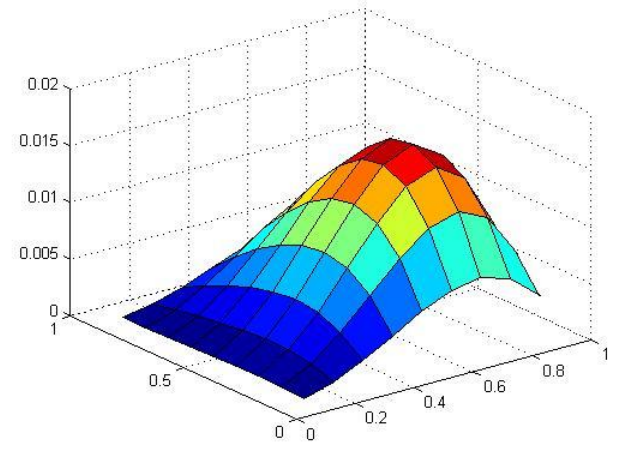

Figure 8- The Corresponding state

(II) In the FWM: the optimal control and corresponding state are given after 60 iterations. The results are: $G_{0}\left(\omega^{s}\right)=7.8110 \mathrm{e}-06, \varrho_{s}=9.7 \mathrm{e}-03$, and $\varepsilon_{s}=9.8 \mathrm{e}-03$

The following figures show the optimal control and its corresponding state at $=0.5$.

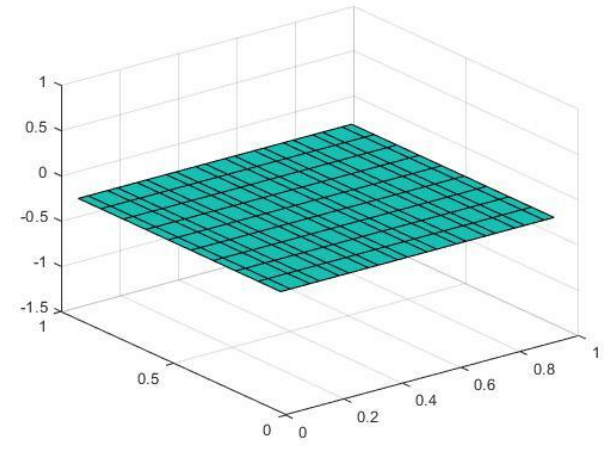

Figure 9- The optimal control

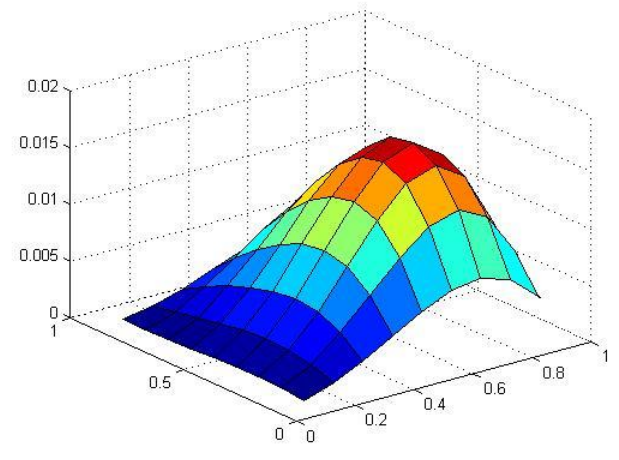

Figure 10- The Corresponding state

7.2 Example: Consider the following CCOCP governed by the VCNLHBVP:

$$
\begin{aligned}
& \psi_{t t}-\frac{\partial}{\partial y}\left[(y+1) \frac{\partial \psi}{\partial y}\right]-\frac{\partial}{\partial z}\left[(z+1) \frac{\partial \psi}{\partial z}\right]+\psi=g(\vec{x}, t)+\sin (\psi)-\sin \left(\psi_{d}\right)+\omega-\omega_{d}, \quad \forall(\vec{x}, t) \in \\
& \rho, E=[0,1], \mathrm{K}=[0,1] \times[0,1], \vec{x}=(y, z)
\end{aligned}
$$


$\psi(\vec{x}, t)=0, \forall(\vec{x}, t) \in \partial \rho=\partial \mathrm{K} \times[0, T]$.

$\psi(\vec{x}, 0)=0$, and $\psi_{t}(\vec{x}, 0)=y z(1-y)(1-z), \forall \vec{x} \in K$

where $g(\vec{x}, t)=y^{2} \sin (t)(y-1)(z-1)-y z \sin (t)(z-1)-y \sin (t)(y-1)(z-1)-$ $2 y \sin (t)(y-1)(z+1)-z \sin (t)(y-1)(z-1)-2 z \sin (t)(y+1)(z-1)-$ $y z \sin (t)(y-1)-y z \sin (t)(y-1)(z-1)$

The control constraint is $U=[-6,5]$ and the cost functional (5), with $\psi_{d}(\vec{x}, t)=y z(1-y)(1-z) \sin (t), \forall(\vec{x}, t) \in \rho$, and $\omega_{d}(\vec{x}, t)= \begin{cases}3.25 & , \text { for } 0 \leq t<0.5 \\ -3 & , \text { for } 0.5 \leq t<1\end{cases}$

with the initial control

$$
\omega_{0}(\vec{x}, t)=\left\{\begin{array}{cl}
3.35 & , 0 \leq t<0.2 \\
2.25 & , 0.2 \leq t<0.5 \\
-1 & , 0.5 \leq t<0.7 \\
2.45 & , 0.7 \leq t<1
\end{array}, \forall(\vec{x}, t) \in \rho .\right.
$$

First, depending on the above initial control and its corresponding state, the following results are obtained according to the optimization methods with ARSO.

(I) In the GM: the optimal control and corresponding state are obtained after 10 iterations. The results are: $G_{0}\left(\omega^{s}\right)=6.6397 \mathrm{e}-06, \varrho_{s}=5.8 \mathrm{e}-03$, and $\varepsilon_{s}=5.5014 \mathrm{e}-04$.

The optimal control and its corresponding state at $t=0.5$ are shown by the following figure.

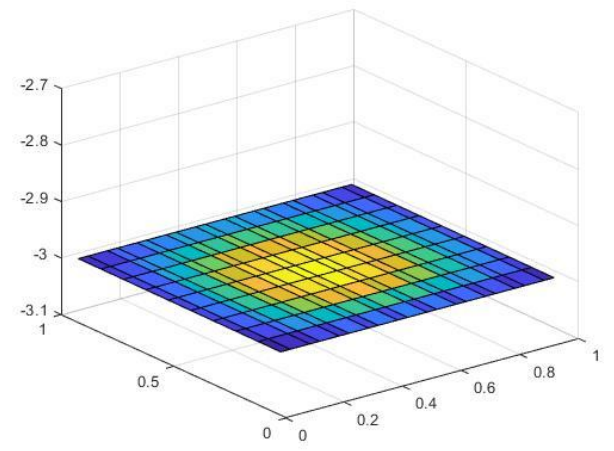

Figure 11- The optimal control

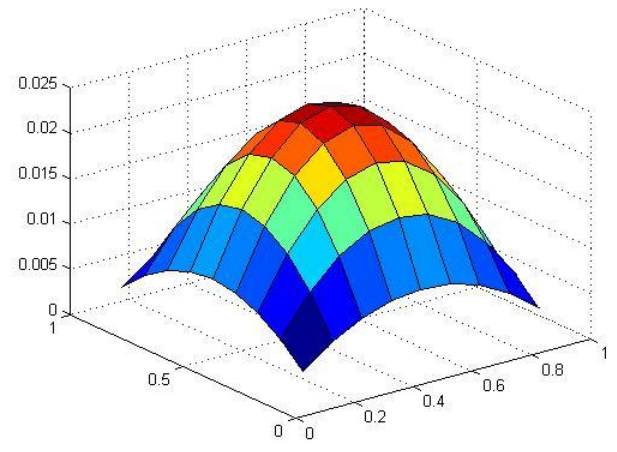

Figure 12- The Corresponding state

(II) In the FWM: the optimal control and corresponding state are obtained after 80 iterations._The results are $: G_{0}\left(\omega^{s}\right)=7.8843 \mathrm{e}-06, \varrho_{s}=6.1 \mathrm{e}-03$, and $\varepsilon_{s}=4.1 \mathrm{e}-03$.

The following figures show the optimal control and its corresponding state.

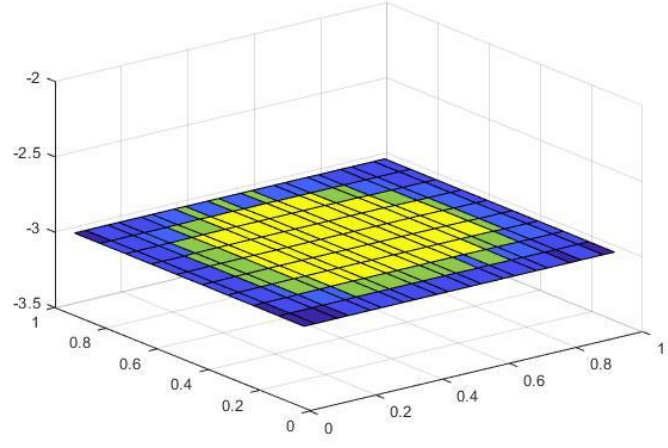

Figure 13- The optimal control

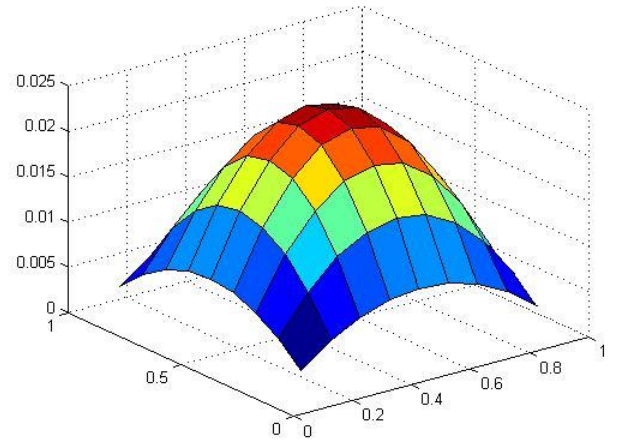

Figure 14- The Corresponding state

(III) In the GPM: the optimal control and corresponding state are obtained after 7 iterations The results are: $G_{0}\left(\omega^{s}\right)=6.6369 \mathrm{e}-06, \varrho_{s}=5.8 \mathrm{e}-03$, and $\varepsilon_{s}=3.9563 \mathrm{e}-04$.

The following figures show the optimal control and its corresponding state. 


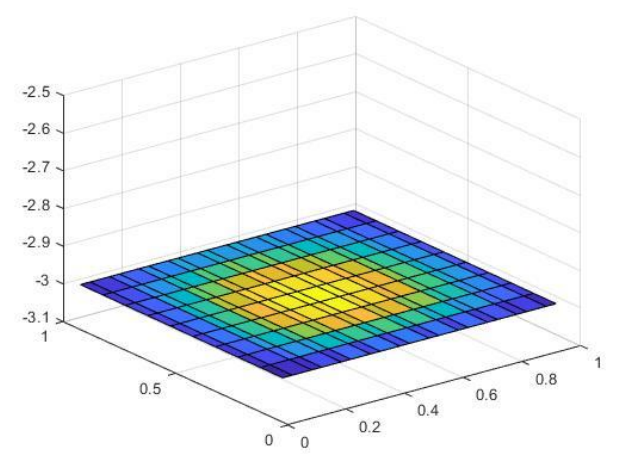

Figure 15- The optimal control

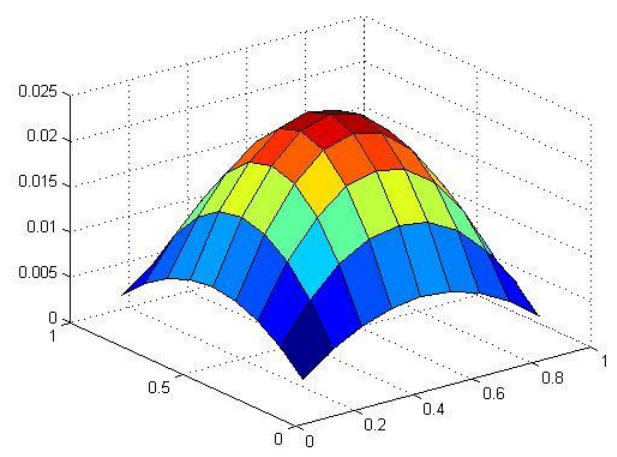

Figure 16- The Corresponding state

Second, the following results are obtained by using the optimization methods with OPSO:

(I) In the GM and GPM: the optimal control and corresponding state are given after 4 iterations, with the results in this case are: $G_{0}\left(\omega^{s}\right)=6.6890 \mathrm{e}-06, \varrho_{s}=5.7 \mathrm{e}-03$, and $\varepsilon_{s}=3.6205 \mathrm{e}-04$.

The optimal control and its corresponding state are shown by the following figures.

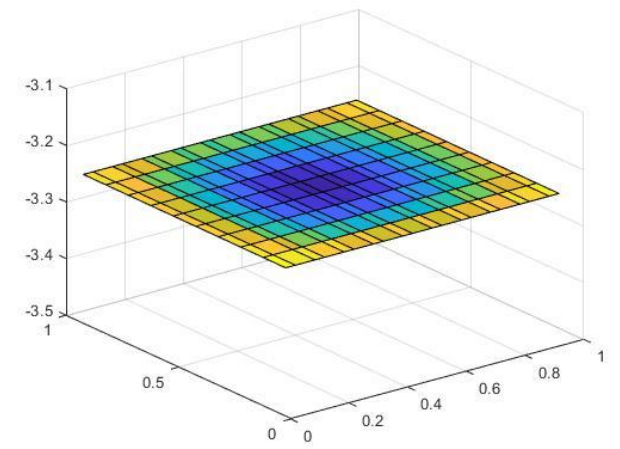

Figure 17- The optimal control

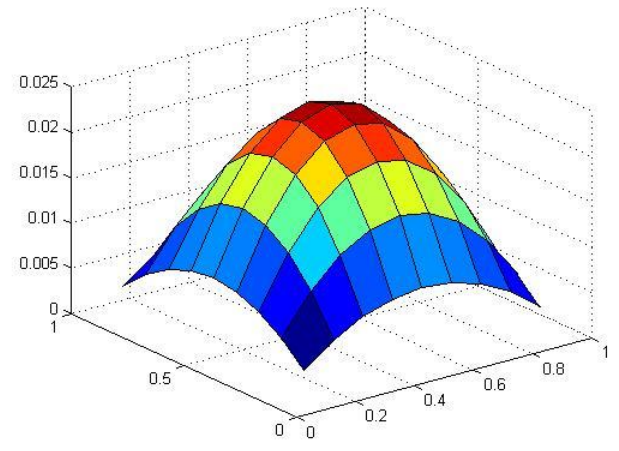

Figure 18- The Corresponding state

(II) In the FWM: the optimal control and corresponding state are given after 42 iterations. The results are: $G_{0}\left(\omega^{s}\right)=1.0879 \mathrm{e}-05, \varrho_{s}=6.7 \mathrm{e}-03$, and $\varepsilon_{s}=9.0 \mathrm{e}-03$.

The following figures show the optimal control and its corresponding state at $t=0.5$.

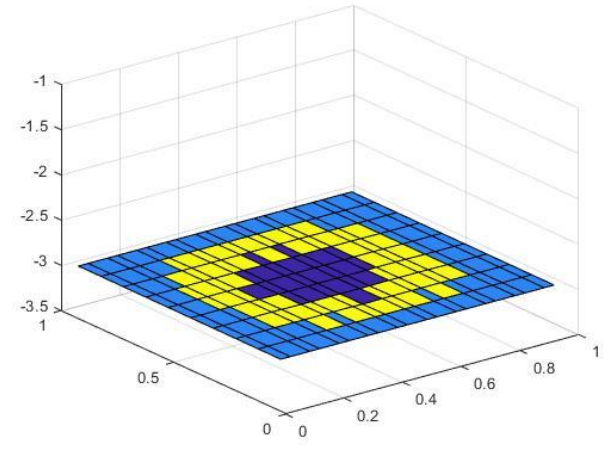

Figure 19- The optimal control

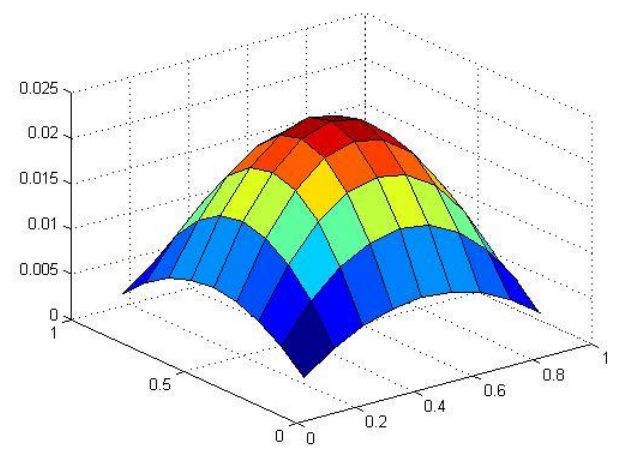

Figure 20- The Corresponding state

Conclusions: The GFEM-IFDS is applied to solve the DWF of VCNLHBVP as well as the DAWF to find the NS, using thestep length of the space variable $h=0.1$ and step length of the time variable $\Delta t=0.05$, along with the parameters $(b=0.5, c=0.5$ and $\mu=0.5)$ in the ARSO. From the results of the two examples above we can point out the following conclusions:

(I) The GFEM-IFDS (according to the use of the values $h$ and $\Delta t$ ) is an accurate, appropriate and fast method to solve the DWF and DAWF. 
(II) The results obtained by applying the GPM with ARSO are better than those obtained by using the GM On the other hand, the results obtained by these two methods are better and faster than those obtained by applying the FWM with ARSO.

(III) The results obtained by the GPM and GM with OPSO method are better and faster than those obtained by using the FWM with the OPSO method.

(IV) For the OPSO and the ARSO, which were used inside the GPM, although the GM method was faster than the FWM one, $i$ it cannot be used in general, since it is only suitable for a quadratic cost function.

Acknowledgement: We wish to express our sincere thanks to the University of Mustansiriyah as well as the College of Science- Department of Mathematics for providing a pleasurableand stimulating environment to carry out the research and for their timely support.

\section{References}

1. Kunisch, K., Pieper, K. and Rund, A. 2016. Time optimal control for a reaction diffusion system arising in cardiac electrophysiology - A monolithic approach. ESAIM Mathematical Modelling and Numerical Analysis, 50(2): 381-414.

2. Wang Y., Luo, X., Yang, Y. and Cui, H. 2016. Optimal control of two dimensional parabolic partial differential equations with application to steel billets cooling in continuous casting secondary cooling zone. Optimal Control Applications and methods, 37(6): 1314-1328.

3. Chryssoverghi, I. 2007. Mixed Discretization-Optimization Methods for Nonlinear Elliptic Optimal Control Problems. LNCE. Springer (Heidelberg), 4310: 287-295.

4. Chryssoverghi, I. 2006. Discretization Methods for Similinear Parabolic Optimal Control Problems. International Journal of Numerical and Modeling, 3(02): 437-457.

5. Toyoğlu, F. 2018. On the Solution of a Optimal Control Problem for a Hyperbolic System. Applied and Computational Mathematics, 7(3): 75-82.

6. Al-Hawasy, J. A. and Al-Rawdanee E.H. 2018. Numerical Solution for Classical Optimal Control Problem Governing by Hyperbolic Partial Differential Equation via Galerkin Finite Element with Gradient and Frank Wolfe Methods. The $2^{\text {nd }}$ Inter. Conf. for Applied and Pure Math., SICAPM2019, Feb 27-28, Baghdad-Iraq.

7. Al-Hawasy J. A. and Al-Rawdanee E. H. 2019. Numerical Solution for Classical Optimal Control Problem Governing by Hyperbolic Partial Differential Equation Vai Galerkin Finite ElementImplicit method with Gradient Projection Methods. Ibn Al-Haitham Journal for Pure and Applied Science, 32(2): 71-80.

8. Sandily, R. and Kumar, S. 2016. Convergence of Discontinuous Finite Volume Discretizations for a Semilinear Hyperbolic Optimal Problem. International Journal of Numerical Analysis and Modeling, 13(6): 926-950.

9. Al-Rawdanee, E.H. and Al-Hawasy, J.A. 2019. Mixed Methods for Solving Classical Optimal Control Governing by Nonlinear Hyperbolic Boundary Value Problem. Accepted (for puplishing ) in the 1STCAS2019.

10. Al-Rawdanee, E.H. and Al-Hawasy, J. A. 2019. Mixed of Implicit Galerkin -Frank Wolf, Gradient, Gradient Projection Method for Solving Classical Optimal Control Governing by Variable Coefficients linear Hyperbolic Boundary Value Problem, Accepted for publishing in the Iraqi Journal of science 2019.

11. Chryssoverghi, I., Coletsos, J. and Kokkinis, B. 2006. Discretization methods for optimal control problems with state constraints. J. Comput. Appl. Math., 191 (1): 1-31. 